\title{
Transanal Endoscopic Microsurgery for the Treatment of Well-Differentiated Rectal Neuroendocrine Tumors
}

\author{
Hyoung Ran Kim, Woo Yong Lee, Kyung Uk Jung, Hyuk Jun Chung, Chul Joong Kim, Hae-Ran Yun, \\ Yong Beom Cho, Seong Hyeon Yun, Hee Cheol Kim, Ho-Kyung Chun \\ Department of Surgery, Samsung Medical Center, Sungkyunkwan University School of Medicine, Seoul, Korea
}

Purpose: Recently, an increase in well-differentiated rectal neuroendocrine tumors (WRNETs) has been noted. We aimed to evaluate transanal endoscopic microsurgery (TEM) for the treatment of WRNETs.

Methods: Between December 1995 and August 2009, 109 patients with WRNETs underwent TEM. TEM was performed for patients with tumors sizes of up to $20 \mathrm{~mm}$ and without a lymphadenopathy. These patients had been referred from other clinics after having been diagnosed with WRNETs by using a colonoscopic biopsy; they had undergone a failed endoscopic submucosal dissection (ESD) or endoscopic mucosal resection (EMR) and exhibited an involved resection margin and remaining tumor after ESD or EMR, regardless of the distance from the anal verge. This study included 38 patients that had more than three years of follow-up.

Results: The mean age of the patients was $51.3 \pm 11.9$ years, the mean tumor size was $8.0 \pm 3.9 \mathrm{~mm}$, and no morbidity occurred. Thirty-five patients were asymptomatic. TEM was performed after a colonoscopic resection in 13 cases because of a positive resection margin, a residual tumor or a non-lifting lesion. Complete resections were performed in 37 patients; one patient with a positive margin was considered surgically complete. In one patient, liver metastasis and a recurrent mesorectal node occurred after five and 10 years, respectively.

Conclusion: TEM might provide an accessible and effective treatment either as an initial or as an adjunct after a colonoscopic resection for a WRNET.

Keywords: Well-differentiated rectal neuroendocrine tumors; Transanal endoscopic microsurgery; Colonoscopic resection; Treatment

\section{INTRODUCTION}

Well-differentiated rectal neuroendocrine tumors (WRNETs) account for approximately $15 \%$ of all gastrointestinal neuroendocrine tumors and $1.3 \%$ of rectal tumors [1]. Yao et al. [2] reported a four-fold increase in the frequency of neuroendocrine tumors,

Received: December 11, 2011 - Accepted: August 21, 2012

Correspondence to: Woo Yong Lee, M.D.

Department of Surgery, Samsung Medical Center, Sungkyunkwan University School of Medicine, 81 Irwon-ro, Gangnam-gu, Seoul 135-710, Korea

Tel: +82-2-3410-0261, Fax: +82-2-3410-6980

E-mail: Iwy555@skku.edu

(C) 2012 The Korean Society of Coloproctology

This is an open-access article distributed under the terms of the Creative Commons Attribution NonCommercial License (http://creativecommons.org/licenses/by-nc/3.0) which permits unrestricted noncommercial use, distribution, and reproduction in any medium, provided the original work is properly cited. including those of the rectum. This higher frequency may be attributed to the widespread use of sigmoidoscopy and colonoscopy for cancer screening and to refinements in tumor classification [2]. Changes in the frequency, the types of diagnostic methods, and the clinical focus of WRNETs have been associated with a newly-emerged definition used to classify tumors as a malignant disease [3].

Buess et al. [4, 5] introduced transanal endoscopic microsurgery (TEM) in 1983. TEM provides better tumor access than a conventional transanal excision (TAE), which has the limitations of poor visualization and of accessibility to only the lower rectum [6, 7]. It also has the advantage of lower rates of a positive resection margin and recurrence [8]. The purpose of this study was to investigate TEM as a method of treatment for WRNETs and to analyze the clinicopathologic results of TEM for WRNETs. 


\section{METHODS}

Between December 1995 and August 2009, 118 patients were treated for WRNETs; 109 underwent TEM, and a radical resection was performed in 9 patients ( 8 low anterior resections, and 1 abdominoperineal resection) at the Samsung Medical Center. Among the consecutive 109 patients that were treated by TEM, 38 patients ( 25 male, and 13 female) with more than three years of follow-up were analyzed in this study. TEM was performed for patients with tumors sizes of up to $20 \mathrm{~mm}$ and without a lymphadenopathy. These patients had been referred from other clinics after having been diagnosed with WRNETs by using a colonoscopic biopsy; they had undergone a failed endoscopic submucosal dissection (ESD) or endoscopic mucosal resection (EMR) and exhibited an involved resection margin and remaining tumor after ESD or EMR, regardless of the distance from the anal verge. The frequency revealed that WRNETs gradually increased from six cases between 1995 and 1999, to 21 between 2000 and 2004 and to 91 between 2005 and 2009 (Fig. 1).

The instrument used for TEM was manufactured by Rich and Wolf Medical Instruments Corporation (Vernon Hills, IL, USA). The rectoscope had a $4-\mathrm{cm}$ diameter and two different lengths, $12 \mathrm{~cm}$ and $20 \mathrm{~cm}$, that were fixed for use on the operating table by using a Martin arm. An airtight face-piece with four ports sealed by rubber sleeves and caps was attached, and carbon dioxide gas was used. Various endoscopic instruments were used to resect the rectal tumor through the ports. The resected tissue specimens were stained with hematoxylin and eosin.

The demographic features, operation times, postoperative hospital days, follow-up duration, tumor size, distance from the anal verge, tumor-related symptoms and reasons for TEM were analyzed. The first postoperative colonoscopy, abdominopelvic computed tomography (CT) and/or transrectal ultrasonography (TRUS) were performed within six months of the procedure. If the results were non-specific, an annual follow-up was recommended.

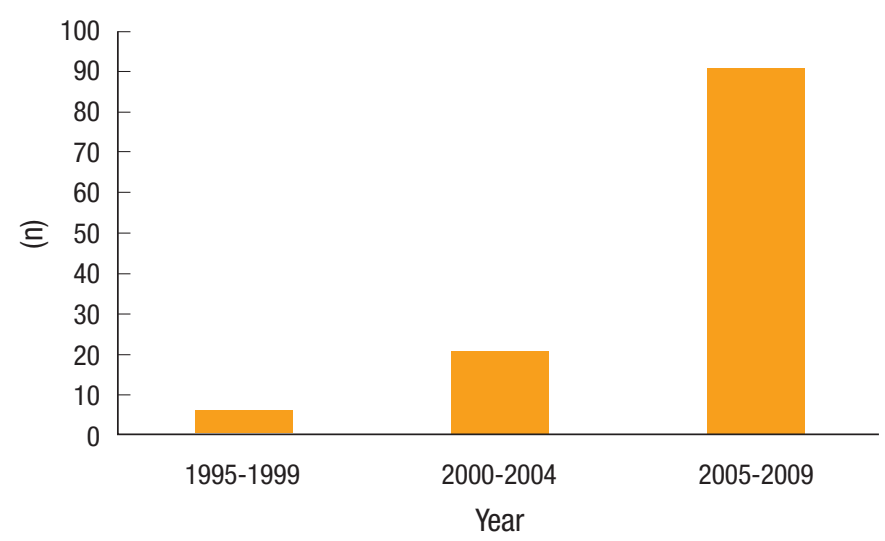

Fig. 1. Incidence of well-differentiated rectal neuroendocrine tumors.

\section{RESULTS}

The patient characteristics are described in Table 1. A preoperative abdominopelvic CT was performed for 16 patients (44.7\%) to investigate distant metastases and perirectal lymphadenopathy. Among 38 patients, 13 had a TEM performed after colonoscopic treatment due to positive resection margins (10 patients, $26.3 \%$ ), remnant lesions ( 2 patients, $5.2 \%$ ) and a non-lifting lesion (1 patient, $2.6 \%$ ). One patient had postoperative urinary difficulty, but recovered with conservative treatment. None of the patients had fecal incontinence during the follow-up.

By the pathologic results, 23 patients had a tumor confined to the submucosa with a negative resection margin. Thirteen patients who had TEM performed after colonoscopic treatment showed no residual tumors with negative resection margins. One woman had a tumor invading the muscle with a negative resection margin; that patient declined additional surgery. One tumor with a pathologically-determined positive margin received no further treatment because the surgeon considered the resection complete. That patient had no recurrence during a more than six-year follow-up.

During a mean follow-up period of 72.4 months, there was one recurrence (2.6\%). One male patient with a $20-\mathrm{mm}$ WRNET who declined radical surgery had bilateral hepatic metastases after five

Table 1. Characteristics of well-differentiated endocrine tumor of rectum

\begin{tabular}{lc}
\hline Characteristic & Value \\
\hline Sex (M:F) & $25: 13$ \\
Age (yr) & $51.3 \pm 11.9$ \\
Operation time (min) & $65.5 \pm 37.9$ \\
Postoperative duration (day) & $1.8 \pm 1.2$ \\
Follow-up duration (mo) & $72.4 \pm 34.7$ \\
Tumor size (mm) & \\
Mean \pm SD & $8.0 \pm 3.9$ \\
$<10$ & $24(63.1)$ \\
$10-19$ & $13(34.2)$ \\
$\geq 20$ & $1(2.7)$ \\
Distance from anal verge (cm) & \\
$<5$ & $5(13.2)$ \\
$5-9.9$ & $24(63.2)$ \\
$\geq 10$ & $6(15.8)$ \\
No description & $3(0.8)$ \\
Symptoms & \\
Screening & $35(92.1)$ \\
Abdominal discomfort & $2(5.3)$ \\
Anal mass & $1(2.6)$ \\
\hline
\end{tabular}

Values are presented as mean \pm SD or number (\%). 
years. This patient underwent a right hemihepatectomy and radiofrequency ablation of a left lobe tumor. Five years after the hepatectomy, mesorectal lymphadenopathy was identified around the previous TEM site, and a low anterior resection was performed. There was no recurrence after one year of follow-up.

\section{DISCUSSION}

Similar to the results of the SEER 17 [2], the results of this study show a recent increase in the frequency of well-differentiated endocrine tumors. This is likely due to an increase in the number of persons undergoing national health colonoscopy screening. According to seventh Union for International Cancer Control revision of staging, gastrointestinal neuroendocrine tumors lesion smaller than $1 \mathrm{~cm}$ are classified as $\mathrm{T} 1$, and all gastrointestinal neuroendocrine tumors are considered malignant [3]. Patients with tumors smaller than $10 \mathrm{~mm}$, representing $80 \%$ of the WRNETs, can be managed by endoscopic resection or TEM [9]. We also had no recurrence in patients with tumors smaller than $20 \mathrm{~mm}$.

TEM has several advantages. It enables the resection, with a sufficient margin, of tumors reaching the upper rectum, and it can be used as an adjunct to failed ESD or EMR procedures. Using TEM, a surgeon can excise upper rectal lesions up to $20 \mathrm{~cm}$ in full thickness, dissect the perirectal area, sample the perirectal lymph nodes, and provide precise suturing of the rectal wall [10]. A resection, in full thickness, facilitates a full investigation of the tumor and lymph nodes and helps to ensure a negative resection margin. Moore et al. [8] compared TEM and TAE for rectal tumors and reported that TEM was more likely than TAE to yield a clear resection margin and a lower recurrence.

Although a conventional endoscopic resection of these tumors avoids the risks of general anesthesia, it is associated with high rates of tumor-positive or uncertain resection margins, of residual tumors $[11,12]$, and of patient discomfort due to the long procedure time [13]. TEM has been reported to take less operation time than ESD $[4,5]$. Even with the cap, an endoscopic submucosal resection leaves a positive resection margin in $14.3 \%$ of the procedures [14].

We investigated the role of TEM as a treatment for only WRNETs. This series from a single institution showed that TEM could obtain negative resection margins during initial surgeries when used as an adjunct for failed endoscopic treatment. However, one patient who was thought to have had a surgically-complete resection was revealed to have had an incomplete resection margin after TEM. That patient had no disease recurrence during the following five years of observation.

The treatment selection and prognosis of patients with WRNETs depends on the tumor size, lymphadenopathy, depth of invasion, angioinvasion and the histological differentiation $[15,16]$. Based on these results and the results of previous studies $[5,10]$, we are refining and recommending our clinical and pathological diagnostic criteria for WRNETs, and we are developing a protocol for pre- and postoperative radiology evaluation, as well as follow-up, based on recent changes in staging. The follow-up and observation period of patients with these tumors must be extended. Prior studies $[15,16]$ have shown that even for tumors smaller than $1 \mathrm{~cm}$ with a low risk for malignancy, a protocol with preoperative evaluation and a follow-up of at least 10 years are essential for optimal treatment and management and for further development of practical guidelines. If the tumor is more than $2 \mathrm{~cm}$ or less than $2 \mathrm{~cm}$ with lymphadenopathy on abdominopelvic CT or magnetic resonance imaging and/or additional risk factors exist, a radical resection should be performed. In cases of tumors less than $1 \mathrm{~cm}$ or between 1 to $2 \mathrm{~cm}$ without lymphadenopathy, a TEM may be considered for the initial procedure (Fig. 2). If the tumor is less than $2 \mathrm{~cm}$ and a TEM is performed, a follow-up with sigmoidoscopy, abdominopelvic CT or TRUS, and chest CT in six months should be carried out. If the results of these studies are non-specific, the follow-up should be yearly. If the tumor treated by radical resection is a WRNET, the scheduled follow-up should be the same as that for a rectal adenocarcinoma.

The limitations of this study include the following. First, WRNETs were generally considered to be benign before the adoption of revised recommendations. As a result, fewer than half of the patients received preoperative radiological evaluations, and there was insufficient information on the risk factors associated with the histological frequency of differentiation, Ki-67 expression and the depth of invasion to assess the patient's prognosis. Second, although recurrence and metastasis were not observed for tumors smaller than $2 \mathrm{~cm}$, the follow-up may have been too short to reveal all of the features associated with these well-differentiated neuroendocrine tumors.

In conclusion, TEM might provide an accessible and effective treatment for WRNETs smaller than $2 \mathrm{~cm}$ either as an initial treatment or adjunct after a colonoscopic resection. Surgically, TEM may achieve an adequate resection margin and full-thickness re-

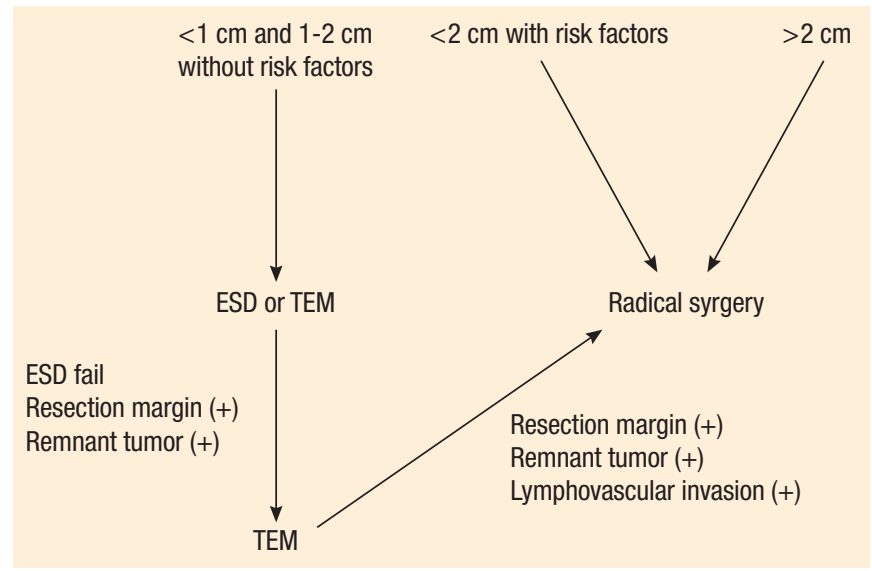

Fig. 2. Our algorithm for the treatment of well-differentiated rectal neuroendocrine tumors.

ESD, endoscopic submucosal dissection, TEM, transanal endoscopic microsurgery. 
section without severe complications.

\section{CONFLICT OF INTEREST}

No potential conflict of interest relevant to this article was reported.

\section{REFERENCES}

1. Fahy BN, Tang LH, Klimstra D, Wong WD, Guillem JG, Paty PB, et al. Carcinoid of the rectum risk stratification (CaRRs): a strategy for preoperative outcome assessment. Ann Surg Oncol 2007; 14:1735-43.

2. Yao JC, Hassan M, Phan A, Dagohoy C, Leary C, Mares JE, et al. One hundred years after "carcinoid": epidemiology of and prognostic factors for neuroendocrine tumors in 35,825 cases in the United States. J Clin Oncol 2008;26:3063-72.

3. Edge SB, Byrd DR, Compton CC, Fritz AG, Greene FL, Trotti A. AJCC cancer staging manual. 7th ed. New York: Springer; 2010.

4. Buess G, Theiss R, Gunther M, Hutterer F, Pichlmaier H. Endoscopic surgery in the rectum. Endoscopy 1985;17:31-5.

5. Buess G, Kipfmuller K, Naruhn M, Braunstein S, Junginger T. Endoscopic microsurgery of rectal tumors. Endoscopy 1987;19 Suppl 1:38-42.

6. Sakamoto GD, MacKeigan JM, Senagore AJ. Transanal excision of large, rectal villous adenomas. Dis Colon Rectum 1991;34:880-5.

7. Mellgren A, Sirivongs P, Rothenberger DA, Madoff RD, GarciaAguilar J. Is local excision adequate therapy for early rectal cancer? Dis Colon Rectum 2000;43:1064-71.

8. Moore JS, Cataldo PA, Osler T, Hyman NH. Transanal endoscopic microsurgery is more effective than traditional transanal exci- sion for resection of rectal masses. Dis Colon Rectum 2008;51: 1026-30.

9. Modlin IM, Kidd M, Latich I, Zikusoka MN, Shapiro MD. Current status of gastrointestinal carcinoids. Gastroenterology 2005; 128:1717-51.

10. Saclarides TJ, Smith L, Ko ST, Orkin B, Buess G. Transanal endoscopic microsurgery. Dis Colon Rectum 1992;35:1183-91.

11. Matsui K, Iwase T, Kitagawa M. Small, polypoid-appearing carcinoid tumors of the rectum: clinicopathologic study of 16 cases and effectiveness of endoscopic treatment. Am J Gastroenterol 1993;88:1949-53.

12. Shirouzu K, Isomoto $H$, Kakegawa T, Morimatsu M. Treatment of rectal carcinoid tumors. Am J Surg 1990;160:262-5.

13. Tominaga K, Nakanishi Y, Nimura S, Yoshimura K, Sakai Y, Shimoda T. Predictive histopathologic factors for lymph node metastasis in patients with nonpedunculated submucosal invasive colorectal carcinoma. Dis Colon Rectum 2005;48:92-100.

14. Sohn DK, Han KS, Hong CW, Chang HJ, Jeong SY, Park JG. Selection of cap size in endoscopic submucosal resection with cap aspiration for rectal carcinoid tumors. J Laparoendosc Adv Surg Tech A 2008;18:815-8.

15. Arnold R. Endocrine tumours of the gastrointestinal tract. Introduction: definition, historical aspects, classification, staging, prognosis and therapeutic options. Best Pract Res Clin Gastroenterol 2005;19:491-505.

16. Ramage JK, Goretzki PE, Manfredi R, Komminoth P, Ferone D, Hyrdel R, et al. Consensus guidelines for the management of patients with digestive neuroendocrine tumours: well-differentiated colon and rectum tumour/carcinoma. Neuroendocrinology 2008; 87:31-9. 